\title{
Continuous or Intermittent? Which Regimen of Enteral Nutrition is Better for Acute Stroke Patients? a Systematic Review and Meta-Analysis
}

\author{
Giovanni Di Paolo*, Elizabeth Twomlow, Fahmy WF Hanna, Adam D Farmer, Joanne Lancaster, Julius Sim and \\ Christine Roffe
}

Royal Stoke University Hospital, The Guy Hilton Research Centre, United Kingdom

*Corresponding author: Giovanni Di Paolo, Guy Hilton Research Centre, Royal Stoke University Hospital, United Kingdom

\begin{abstract}
Background and purpose: Enteral nutrition via nasogastric tube in acute stroke patients with dysphagia is an important determinant of patient outcomes. It is unclear whether intermittent or continuous feeding is more efficacious. The aim of this review is to examine the current evidence comparing the effectiveness of intermittent versus continuous feeding in stroke patients in terms of nutritional status, gastrointestinal intolerance and other complications.

Methods: A systematic review of randomized controlled studies comparing intermittent with continuous nasogastric feeding in acute stroke patients was conducted in accordance with PRISMA (Preferred Reporting Items for Systematic Reviews and Metaanalyses) guidance using predefined search terms. The search was conducted in MEDLINE and EMBASE up to 1st March 2019. Two independent reviewers assessed study quality using the Joanna Briggs Institute Critical Appraisal Tool. Meta-analyses were conducted, where appropriate, using a random-effects model to pool risk ratio with corresponding $95 \% \mathrm{CI}$.

Results: Three studies including a total of 184 patients were identified. All three were medium to low quality. The definition of intermittent enteral nutrition within each study varied considerably in terms of volume, rate and mode of delivery. Achievement of nutritional targets was the same for both feeding patterns in the one study it was reported. Only aspiration pneumonia and diarrhea were measured by all three studies. There was no significant difference in the incidence of aspiration pneumonia (RR $0.91,95 \% \mathrm{CI}$ $0.53-1.57, \mathrm{p}=0.74, \mathrm{I} 2=50 \%$ ) and diarrhea (RR $1.74,95 \% \mathrm{CI} 0.70-4.30, \mathrm{p}=0.23, \mathrm{I} 2=42 \%$ ) between the two patterns of feeding. Other outcomes including, vomiting, gastric retention, mortality, pre-albumin and nasogastric tube complications showed no significant differences.

Conclusion: There is very little and low-quality evidence to inform patterns of enteral feeding after stroke. The available evidence shows no significant difference in nutritional achievement and complications between intermittent and continuous nasogastric tube feeding in acute stroke patients.
\end{abstract}

Keywords: Stroke; Enteral; Nutrition; Nasogastric; Dysphagia

\section{Background}

Dysphagia occurs in up to $50 \%$ of patients following a stroke [14] and increases the risk of pneumonia almost ten-fold [5]. Strokerelated pneumonia is associated with longer length of hospital stay, worse levels of disability and increased mortality [6-9]. In most dysphagic patients, adaptation of the consistency of diet and fluids is sufficient to ensure that the swallow is safe. However, in a small proportion insertion of a Nasogastric Tube (NGT) is required to ensure safe and adequate nutrition. Despite this, more than twothirds of NGT-fed stroke patients still develop pneumonia [10]
Gastric dysmotility is a well-documented phenomenon that occurs in critically ill patients, including acute stroke patients, whereby incomplete gastric emptying results in stasis, heightening the risk of reflux and aspiration of gastric contents [10-13]. NGT bolus feeding was first described by Morrison et al. [14] in 1895 for children with Diphtheria, who received 6-ounce bolus feeds 3 times a day via NGT. However, it wasn't until 1910s when Morgan et al. [15] and Jones et al. [16] began administering their enteral feeds "drop by drop" rather than as a bolus. Contemporaneously, the 
regimen most frequently used in most patients requiring enteral feeding is continuous (i.e. low volume pumped feed lasting 16-24 hours without interruption). However, recent attention has been afforded to examining whether a discontinuous feeding strategy often described as either intermittent or bolus (i.e. high volume of feed administered over a short period multiple times a day) - could reduce patients' risk of pneumonia and achieve better nutrition and digestive tolerance.

Intermittent feeding reflects normal human feeding patterns more closely than continuous feeding. A period of fasting interrupted by the ingestion of a discrete meal causes gastric distension and subsequent stimulation of gut motility, secretion of digestive enzymes and metabolic responses to nutrient loading [1718]. This physiological gastrointestinal response to intermittent feeding has been demonstrated in healthy adults, neonates and intensive care populations [17-20]. While there are good theoretical reasons to assume that intermittent feeding is more physiological, most stroke patients in the UK receive nasogastric feeding continuously, as there are concerns that intermittent feeding may be less well tolerated. Guidance and practice relating to enteral feeding after stroke differs between countries; with the American Heart Association [21] and the Royal College of Physicians [22] not addressing the issue, Australian Guidelines allowing for both options [23] and intermittent feeding described as "traditional" in China [24]. The aim of this systematic review is to determine whether there are differences in the achievement of adequate nutrition, gastrointestinal tolerance, and metabolic stability between intermittent and continuous nasogastric feeding.

\section{Methods}

This systematic review and meta-analysis were prepared according to PRISMA (Preferred Reporting Items for Systematic Reviews and Meta-Analyses) guidelines [25].

\section{Criteria for Considering Studies for this Review}

The inclusion criteria for this review were:

a. Population: Acute stroke patients aged 18 or more with a nasogastric tube receiving enteral nutrition

b. Intervention: Intermittent enteral nutrition: by bolus, gravity systems or infusion pump several times a day with a rest between feeds

c. Control: Continuous enteral nutrition: with gravity systems or infusion pumps, without interruption for a minimum period of 12 hours/day

d. Outcomes: Nutritional status, aspiration pneumonia, diarrhea, vomiting, gastric distension, gastric retention, hyperglycemia, pre-albumin, mortality, length of stay, and NGT complications

e. Study Design: Randomized controlled trials or pseudo-randomised controlled trials (a study without true randomisation) that compared continuous and intermittent enteral feeding methods.

\section{Search Strategy}

A literature search was performed using MEDLINE (1966 $1^{\text {st }}$ March 2019) and EMBASE (1974- $1^{\text {st }}$ March 2019). Studies were searched for using the terms enteral, nutrition, nasogastric, gastrointestinal, feeding as Medical Subject Heading (MeSH) and free text terms. These were combined with the set operator "AND" with following terms: intermittent, continuous as both MeSH and free text terms. Publications were restricted to those studying adult populations, defined as greater than 18 years old, with a documented diagnosis of stroke according to accepted international criteria [26]. This search strategy is described in Appendix 1. The reference lists of all eligible studies that were identified were also comprehensively searched for studies not identified using the initial search strategy. This search was performed independently by two reviewers.

\section{Selection of studies}

Two reviewers (GDP and ET) assessed the studies independently for inclusion using the title and abstract. In cases where relevance could not be determined solely from the abstract, the full text was consulted. Any disagreements were resolved by consensus with a third reviewer (CR).

\section{Data extraction and management}

Data extraction was done manually by two reviewers (GDP and ET). Differences were discussed and adjudicated in faceto-face meetings. Foreign language papers were translated, and descriptions of each study were derived. This included authors, year of publication, type of participant, location, study design, sample size, age and gender of participants, exclusion criteria, when feeding was started, monitoring period, nasogastric tube size, type of feed and definitions of each intervention. In addition, data was extracted for definition and results of each outcome from all studies.

\section{Assessment of risk of bias in included studies}

Methodological quality of the studies was assessed using the Joanna Briggs Institute Meta-Analysis of Statistics Assessment and Review Instrument (MAStARI) Critical Appraisal tool for experimental studies [27].

\section{Data synthesis}

The studies presented in this review all fitted the conceptual definitions of intermittent and continuous enteral nutrition, as outlined in the inclusion criteria. However, there were differences in the volume, rate and temperature of nutrient delivered. In addition, two of the studies did not use true randomisation. Taking into consideration these limitations, a meta-analysis has been carried out with the outcome's diarrhea and aspiration pneumonia, as these were the outcomes assessed by all studies. Narrative synthesis was used where outcomes did not allow meta-analysis. The meta-analysis was performed using Review Manager (RevMan) Version 5.3. Copenhagen: The Nordic Cochrane Centre, The Cochrane Collaboration, 2014. Data was extracted from all three studies for the outcome's diarrhea and aspiration pneumonia. We 
calculated risk ratios (RR) and 95\% CIs using the Mantel-Haenszel model. Statistical heterogeneity among trials was assessed by the I2 test, with $\mathrm{I} 2>50$ representing possible substantial heterogeneity.

\section{Results}

\section{Study selection}

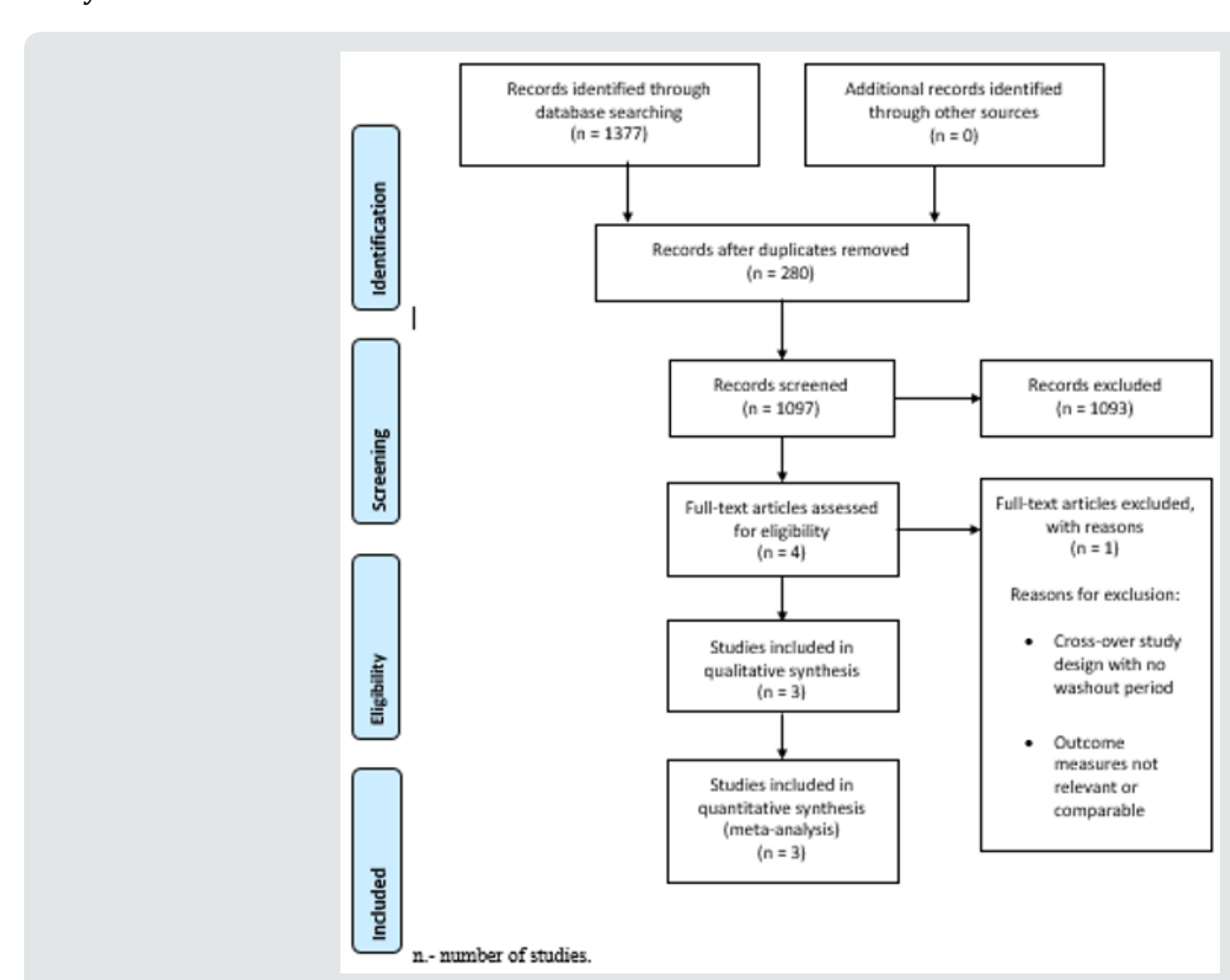

Figure 1: PRISMA flowchart for study selection process.

The PRISMA flow-chart for study selection is shown in Figure 1. Following the removal of duplicates the number of potentially relevant studies identified from this search was 1,377. Four studies met the criteria of relevance and no studies were added following a secondary manual search. On review of the full-texts, one study [28] was excluded due to a cross-over study design with no washout period and the outcomes reported were not clinically relevant to this review. Three studies [25] [29,30] remained including a total

of 184 patients.

\section{Study characteristics} in Appendix 2.
The meta-analysis was performed with a random-effects model irrespective of the level of heterogeneity as the included trials varied considerably in a number of methodological features.

Table 1 shows the characteristics of the included studies and patients. Two studies were conducted in China [24,25,29] and one in Turkey [30]. Population sizes (52-69) and age (mean 61-69 years) were similar in all three. A summary of the studies is given 


\begin{tabular}{|c|c|c|c|}
\hline 离 & 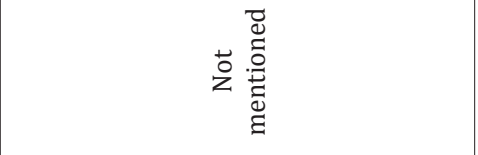 & 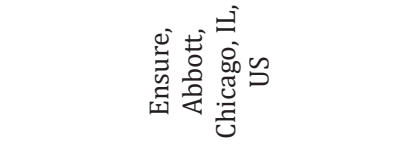 & 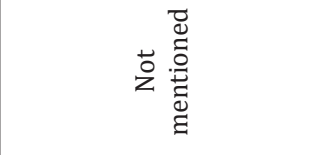 \\
\hline 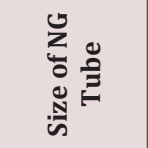 & 艺 & 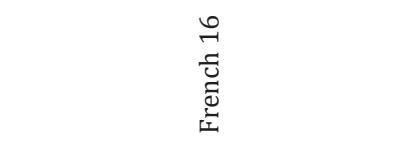 & 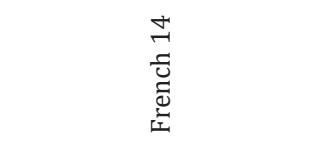 \\
\hline 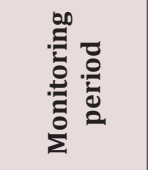 & 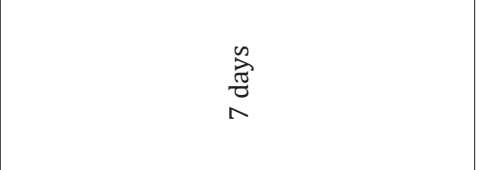 & 疍 & $\begin{array}{l}\text { n } \\
\frac{0}{0} \\
0 \\
-1\end{array}$ \\
\hline 离 & 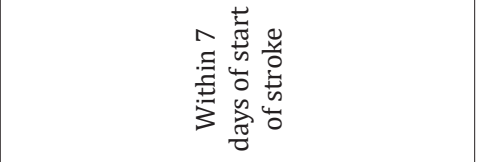 & 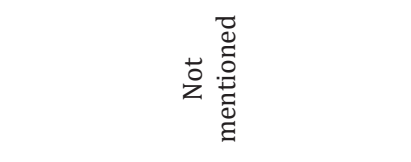 & zे \\
\hline 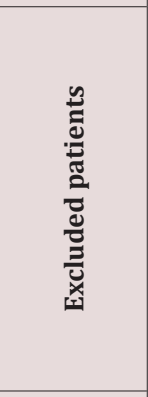 & 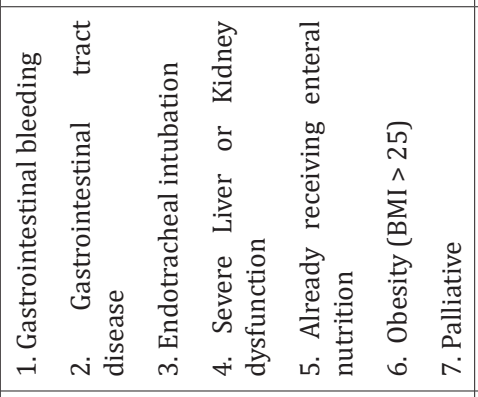 &  & 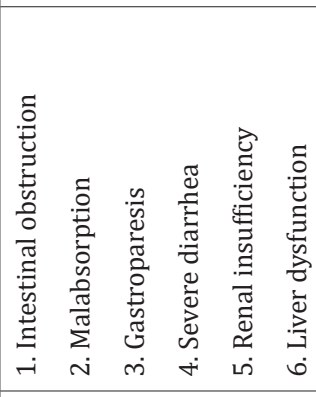 \\
\hline 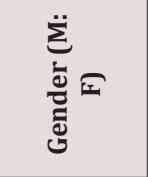 & 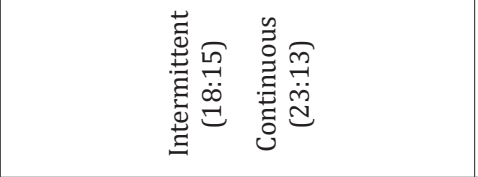 & 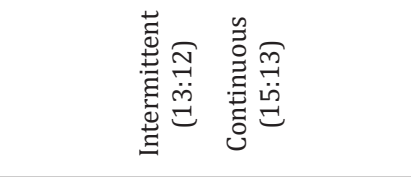 & 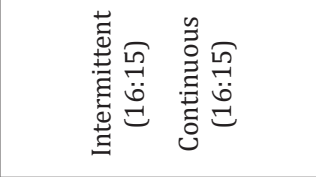 \\
\hline 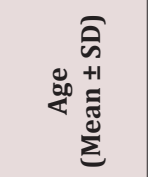 & 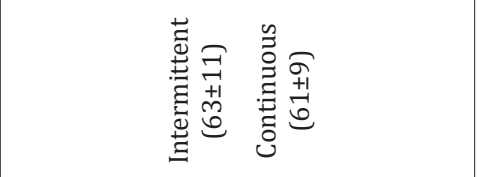 & 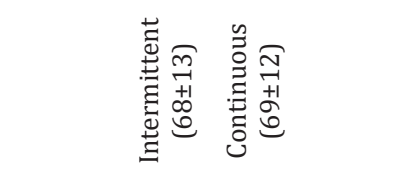 & 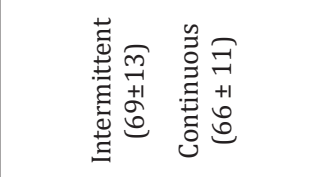 \\
\hline 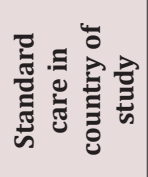 & 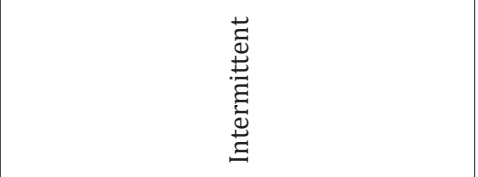 &  & 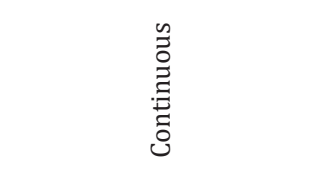 \\
\hline 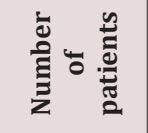 & వి & กี & $\widetilde{్}$ \\
\hline 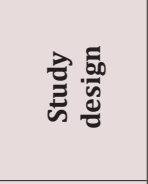 & 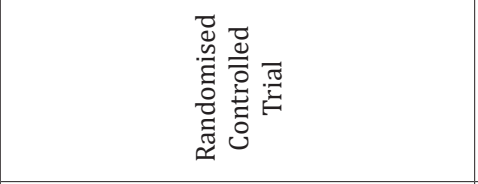 & 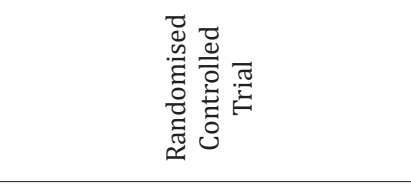 & 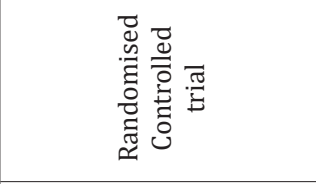 \\
\hline 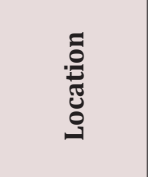 & 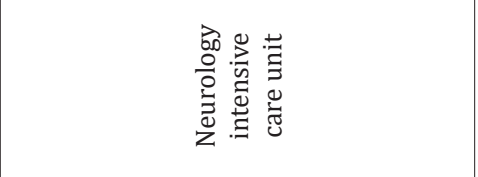 &  & 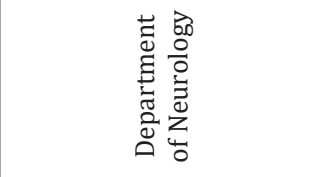 \\
\hline 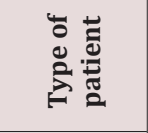 & 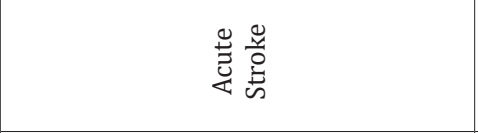 & 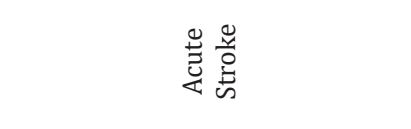 & 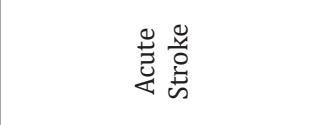 \\
\hline 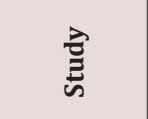 & 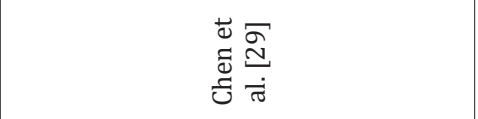 & 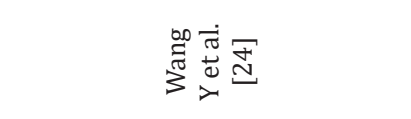 &  \\
\hline
\end{tabular}




\section{Risk of bias and quality of the evidence}

Appendix 3 shows details of the quality assessment with moderate risk of bias (9/13 quality criteria fulfilled by Wang, and 6/13 by Chen and Gungor respectively). Only one study (Wang) was truly randomized (random numbers table), while Chen used alternate assignment for allocation of treatment groups, and Gungor randomized patients into two groups taking into account the age and gender, with no more detail has been given regarding how they randomised. Wang randomised patients using a random number table. Blinding of participants and assessors was not feasible due to the nature of the intervention and the outcomes measured. Only Chen commented on removal of patients from the study for clinical reasons. Four patients were excluded within three days of enrolment because of left ventricular failure, cerebral herniation, gastrointestinal haemorrhage, and respiratory failure respectively. It was not reported whether these patients were included in an intention to treat analysis. The other two studies did not refer to removal of patients after allocation to treatment groups. Chen was the most comprehensive in demonstrating similar baseline characteristics using age, gender, Glasgow Coma Scale, [31] the Acute Physiology, Age, Chronic Health EvaluationII scale, [32] the National Institutes for Health Stroke Scale, [22] and the Barthel index [33]. Gungor used age, gender and a stroke subscale, and Wang only used age, gender and the Glasgow Coma Scale. The only outcome measure that is likely to be unreliable is the assessment of gastric distension in Wang's study. This was ascertained by palpation combined with measurement of abdominal circumference, a method which is not validated and has no defined criteria.

\section{Delivery of the feeds}

Feed was given via wide bore NGTs (16 and $14 \mathrm{~F}$ for Wang and Gungor respectively). Details of administration given in Appendix 4. Continuous enteral nutrition was delivered via an infusion pump in all three studies, initially at less than $50 \mathrm{ml} / \mathrm{h}$ increasing to 75-100 $\mathrm{ml} / \mathrm{h}$ as tolerated. Gungor started at a slower rate $(10 \mathrm{ml} / \mathrm{h})$ than the other two studies and increased feeding rates more gradually. Two studies (Wang and Gungor) continued feeding overnight without a period of rest, while Chen discontinued the feed for a period of 7 hours overnight. Intermittent regimens were considerably different between the studies. Wang delivered each feed (200-300 ml) over 10-15 minutes at a rate of $800-1800 \mathrm{ml} / \mathrm{h}$, while Gungor infused a smaller volume of feed (120 ml) over a longer period of time (30-60 $\mathrm{min}$ ) at a much slower rate of 300-600 $\mathrm{ml} / \mathrm{h}$. Wang administered the feed manually with a $50 \mathrm{ml}$ syringe, which may have resulted in an even quicker administration time by the nurses than appreciated by the assessors of the study.

\section{Outcomes}

The effects of intermittent and continuous feeding on clinically relevant outcomes are given in Table 2. Definitions for key outcomes are detailed in Appendix 5.

Table 2: Comparative effects of intermittent and continuous enteral nutrition on clinically relevant outcomes. $\mathrm{g} / \mathrm{L}$ : grams per litre.

\begin{tabular}{|c|c|c|c|}
\hline & Intermittent & Continuous & Difference \\
\hline $\begin{array}{l}\text { Nutrition (\% daily calorific requirements achieved) } \\
\text { Chen [29] }\end{array}$ & $\begin{array}{c}84.80 \% \\
\text { (95\% CI - } 75.7 \% \text { to } 93.9 \%) \\
\end{array}$ & $\begin{array}{c}93.90 \% \\
\text { (95\% CI - } 77.9 \% \text { to } 99.8 \%)\end{array}$ & $P>0.05$ \\
\hline $\begin{array}{c}\text { Diarrhoea (no. of patients) } \\
\text { Chen [29] } \\
\text { Wang [24] } \\
\text { Gungor [30] }\end{array}$ & $\begin{array}{l}9 / 33(27.3 \%) \\
13 / 25(52.0 \%) \\
7 / 31(22.6 \%)\end{array}$ & $\begin{array}{c}11 / 36(30.6 \%) \\
7 / 28(25.0 \%) \\
1 / 31(3.2 \%)\end{array}$ & $\begin{array}{l}P=0.76 \\
P=0.04 \\
P=0.05\end{array}$ \\
\hline Vomiting (no. of patients) Gungor [30] & $0 / 31(0.0 \%)$ & $0 / 31(0.0 \%)$ & $P=1.00$ \\
\hline Gastric distention (no. of patients) Wang [24] & $16 / 25(64.0 \%)$ & $4 / 28(14.3 \%)$ & $\mathrm{P}<0.01$ \\
\hline $\begin{array}{l}\text { Gastric Retention (no. of patients) } \\
\text { Chen [29] } \\
\text { Gungor [30] }\end{array}$ & $\begin{array}{l}1 / 33(3.0 \%) \\
0 / 31(0.0 \%)\end{array}$ & $\begin{array}{l}1 / 36(2.8 \%) \\
0 / 31(0.0 \%)\end{array}$ & $\begin{array}{l}P=1.00 \\
P=1.00\end{array}$ \\
\hline $\begin{array}{c}\text { Pneumonia (no. of patients) } \\
\text { Chen [29] } \\
\text { Wang [24] } \\
\text { Gungor [30] }\end{array}$ & $\begin{array}{l}11 / 33(33.3 \%) \\
10 / 25(40.0 \%) \\
11 / 31(35.5 \%)\end{array}$ & $\begin{array}{l}21 / 36(58.3 \%) \\
9 / 28(32.1 \%) \\
9 / 31(29.0 \%)\end{array}$ & $\begin{array}{l}P=0.04 \\
P=0.55 \\
P>0.05\end{array}$ \\
\hline Mortality (no. of patients) Gungor [30] & $1 / 31(3.2 \%)$ & $3 / 31(9.7 \%)$ & $\mathrm{P}>0.05$ \\
\hline Length of admission (days) Gungor [30] & 16.8 & 14.1 & $\mathrm{P}>0.05$ \\
\hline Hyperglycaemia (no. of patients) Wang [24] & $11 / 25(44.0 \%)$ & $5 / 28(17.9 \%)$ & $\mathrm{P}=0.04$ \\
\hline Pre-albumin (g/L) Chen [29] & 0.18 & 0.17 & $\mathrm{P}>0.05$ \\
\hline NGT complications (no. of patients) Gungor [30] & $8 / 31(25.8 \%)$ & $7 / 31(22.6 \%)$ & $P>0.05$ \\
\hline
\end{tabular}

\section{Achievement of nutritional targets}

This was only reported in one study [29]. There was no significant difference in achievement of the nutritional target and in levels of pre-albumin between intermittent and continuous feeding. 


\section{Complications of nasogastric feeding}

One of the three studies (Chen) showed a significantly higher incidence $(58.3 \%)$ of pneumonia with continuous feeding than with intermittent feeding (33.3\%), with no difference in the other two studies. Diarrhea was significantly more frequent with intermittent feeding (64.0\% vs. $14.3 \%)$ in Wang, but not in the other two studies. Wang also reported significantly more hyperglycaemia with intermittent feeding. No significant differences were found for vomiting (Gungor), gastric retention (Gungor, Wang), and NGT complications (Gungor).

\section{Other outcomes}

One study (Gungor) reported mortality and length of stay. No significant differences were identified between feeding patterns.

\section{Meta-analysis}

i) Aspiration pneumonia

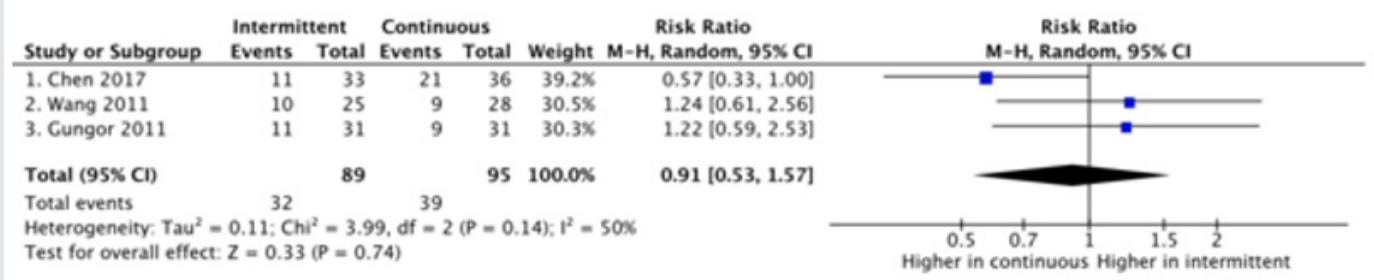

\section{ii) Diarrhea}

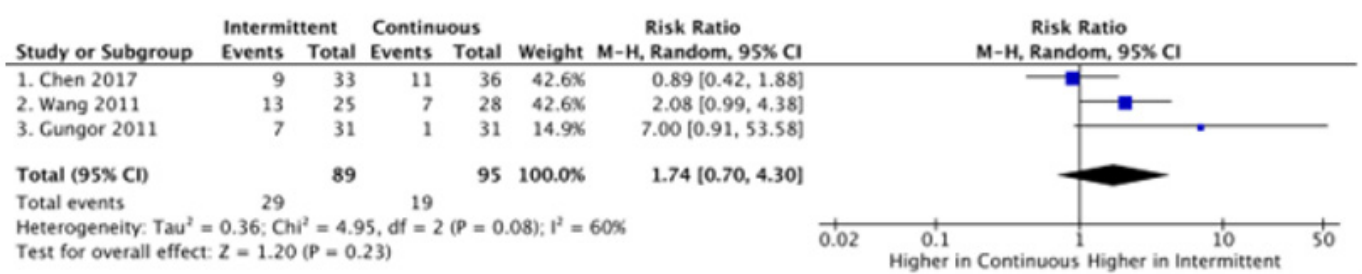

Figure 2: Meta-analysis of intermittent compared with continuous enteral nutrition on the incidence of aspiration pneumonia and diarrhea in acute stroke patients.

CI: Confidence interval; Chi2: Chi-squared test; Tau: Tau test

Only aspiration pneumonia and diarrhea were assessed by all three studies and could be included in the meta-analysis. There was no significant difference between intermittent and continuous feeding in either incidence of aspiration pneumonia (RR 0.91, 95\% CI $0.53-1.57, \mathrm{p}=0.74, \mathrm{I} 2=50 \%$ ) or diarrhea (RR $1.74,95 \%$ CI 0.70 $4.30, p=0.23, I 2=42 \%)$. A funnel plot is not presented here as there were only 3 trials. This is analysis is displayed in Figure 2 .

\section{Discussion}

The systematic review identified three studies comparing intermittent and continuous nasogastric feeding including 184 acute stroke patients. There was no significant difference between feeding regimes for most outcomes in individual studies with the exception of pneumonia, which was higher with continuous feeding in one study [29] and diarrhea, gastric distension and hyperglycemia, which were seen more frequently in another study [24]. The only outcomes which were assessed by all three studies and could be included in the meta-analysis were aspiration pneumonia and diarrhea, neither of which were significantly different in the two feeding regimens. Intermittent feeding would be expected to improve achievement of nutritional goals, as it is closer to normal feeding patterns allowing for more physiological gastrointestinal and metabolic responses. There is insufficient evidence to determine the effect of feeding pattern on the achievement of nutritional goals in this patient group. In the one study [29], where nutritional goals were addressed, no significant difference was found. Studies in intensive care patients found that calorific objectives were more likely to be achieved with intermittent than with continuous enteral nutrition [34,35] and this was confirmed through systematic review [21]. Furthermore, studies examining these two methods of administering enteral nutrition in older adults on general wards also found no discernible difference in the calories achieved [36,37] This was in keeping with the results observed from this review.

Aspiration pneumonia is a major complication of dysphagic stroke and may be affected by the pattern of feeding. Our metaanalysis did not find a significant difference in pneumonia between intermittent and continuous feeding. In all three studies 
the minimum incidence of aspiration pneumonia in acute stroke patients fed by NGT was regardless of intervention. Chen was an outlier with almost twice the incidence of aspiration pneumonia in the continuous group, and this difference might have been due to chance. However, this was the only study to specify that they recruited patients within 7 days of admission, and this could have ensured that patients hadn't had a significantly long starvation period in which gastric dysmotility would have developed. Interestingly, it was the only study which discontinued feeding during the night, a practice usually considered to reduce the risk of pneumonia. Studies of intermittent versus continuous feeding in other settings give mixed results with a reduction of pneumonia with intermittent feeding in intensive care,38 but no difference in older people nursed on general wards [37]. Gastrointestinal tolerance is a major determinant of choice of feeding pattern. There was no significant difference in the incidence of diarrhea in our meta-analysis. Looking at individual studies, Wang consistently reported more gastrointestinal and metabolic adverse effects in the intermittent feeding group than with continuous feeding with a significantly higher incidence of diarrhoea, gastric distension, and hyperglycaemia. While this might have been a chance effect, it could have been due to differences in the delivery of the feeds. They gave intermittent feeding manually via a $50 \mathrm{ml}$ syringe rather than by pump and at a much higher rate (200-300 ml over 10-15 minutes). Wang was the only study to warm their feed to body temperature (37 degrees in the intermittent group and 40 degrees in the continuous group to allow for slower infusion rates).

This would be expected to improve tolerance [39-41] especially with the larger volumes in the intermittent feeding group [42]. Both gastric distention and retention are known to be affected by gastric motility/emptying, which has been shown to be improved by the use of intermittent enteral nutrition in healthy adults[17,18]. However, in intensive care patients, where gastric dysmotility is common, studies have consistently demonstrated no difference between intermittent and continuous nutrition [43-48]. Several previous studies, largely conducted in intensive care, demonstrated that gastrointestinal tolerance was similar with intermittent and continuous enteral nutrition. However, [36] 1992 found a very high frequency of diarrhea in older adults on intermittent compared with continuous feeding ( $96 \%$ v 66\%, p <0.008).36 Not to the same extent, this finding was also reported by Hiebert et al 1981 in adult patients with burns.44 However, in a systematic review in intensive care patients by Martinez 2014 [20] there was no significant difference between intermittent and continuous enteral nutrition with regards to gastrointestinal tolerance. This is corroborated by our results, which has also shown no significant difference in incidence of diarrhoea when comparing intermittent with continuous enteral nutrition.

The measurement of gastric residual volume (GRV) is not standard practice for acute stroke patients admitted in the UK, although it is carried out in patients on intensive care units. Two of the studies used GRV to assess gastric retention as an outcome measure; this will have required large bore NGT (French 1416 as described in Wang and Gungor). These size NGT are not normally required for standard feeding regimens and would have facilitated the faster rates of feeding seen in these studies. Glycaemic responses to feeding were only assessed in one study (Wang), where hyperglycaemia was found to significantly more common with intermittent feeding. In this study, blood glucose was measured every 4 hours and a blood glucose of more than $8.0 \mathrm{mmol} / \mathrm{L}$ was documented as an episode of hyperglycaemia. It has previously been shown that increasing gastric emptying heightens postprandial glycaemic excursions, [49] which is likely to be the case in intermittent feeding. In an of itself, hyperglycaemia potentiates the slowing of gastric emptying [50,51] which is an important factor considering its sequential impact on gastric retention. However, this may not mean that the overall glycaemic control is worse than with continuous feeding, which would be better assessed through 24-hour blood glucose monitoring. The limitations of the review are the small number of studies, the limited number of participants, and the moderate quality of the evidence. There is a risk of bias which was evident when significant findings in individual studies were no longer evident in systematic review. While the interventions and populations where comparable, there were variations in the definition of intermittent and continuous feeding and delivery of feeds which may have accounted for some of the differences observed between individual studies.

\section{Conclusion}

In conclusion, there are only few studies comparing intermittent with continuous feeding in stroke patients, and these are of low quality with small sample sizes. The definitions of intermittent enteral nutrition varied, and the findings were inconsistent. Based on this review, no definitive conclusion can be made as to which method of delivery of nutrition by nasogastric tube is safer and more effective in acute stroke patients. Further research is warranted to address this.

\section{References}

1. Singh SA, Hamdy S (2006) Dysphagia in stroke patients. Postgraduate medical journal 82(968): 383-391.

2. Smithard DG, O Neill PA, Park CL, Morris J (1996) Complications and outcome after acute stroke: Does dysphagia matter? Stroke 27(7): 12001204.

3. Gordon C, Hewer RL, Wade DT (1987) Dysphagia in acute stroke. Br Med J (Clin Res Ed) 295(6595): 411-414.

4. Barer DH (1989) The natural history and functional consequences of dysphagia after hemispheric stroke. Journal of Neurology, Neurosurgery \& Psychiatry 52(2): 236-241.

5. Walter U, Knoblich R, Steinhagen V, Donat M, Benecke R, et al. (2007) Predictors of pneumonia in acute stroke patients admitted to a neurological intensive care unit. Journal of neurology 254(10): 13231329.

6. Heuschmann PU, Kolominsky Rabas PL, Misselwitz B, Hermanek P, Leffmann C, et al. (2004) Predictors of in-hospital mortality and attributable risks of death after ischemic stroke: The German Stroke Registers Study Group. Archives of internal medicine 164(16): 17611768.

7. Wilson RD (2012) Mortality and cost of pneumonia after stroke for different risk groups. Journal of Stroke and Cerebrovascular Diseases 21(1): 61-67.

8. Masrur S, Smith EE, Saver JL, Reeves MJ, Bhatt DL, et al. (2013) Dysphagia screening and hospital-acquired pneumonia in patients with acute 
ischemic stroke: Findings from Get with the Guidelines-Stroke. Journal of Stroke and Cerebrovascular Diseases 22(8): 301-309.

9. Finlayson O, Kapral M, Hall R, Asllani E, Selchen D, et al. (2011) Risk factors, inpatient care, and outcomes of pneumonia after ischemic stroke. Neurology 77(14): 1338-1345.

10. Langdon PC, Lee AH, Binns CW (2009) High incidence of respiratory infections in nil by mouth tube-fed acute ischemic stroke patients. Neuroepidemiology 32(2): 107-113.

11. Schaller BJ, Graf R, Jacobs AH (2006) Pathophysiological changes of the gastrointestinal tract in ischemic stroke. The American journal of gastroenterology 101(7): 1655-1665.

12. Rombeau JL, Takala J (1997) Summary of round table conference: Gut dysfunction in critical illness. Intensive care medicine 23(4): 476-479.

13. Corke C (1999) Gastric emptying in the critically ill patient. Critical Care and Resuscitation 1(1): 39-44.

14. MORRISON WA (1895) The value of the stomach tube in feeding after intubation, based upon twenty-eight cases; also, its use in post diphtheritic paralysis. The Boston Medical and Surgical Journal 132(6): 127-130.

15. Morgan WG (1914) Duodenal Alimentations 1. The American Journal of the Medical Sciences (1827-1924 148(3): 360.

16. Jones CR (1916) Duodenal feeding. SurgGynecolObstet 22: 236-240

17. Bowling TE, Cliff B, Wright JW, Blackshaw PE, Perkins AC, et al. (2008) The effects of bolus and continuous nasogastric feeding on gastro oesophageal reflux and gastric emptying in healthy volunteers: A randomised three way crossover pilot study. Clinical Nutrition 27(4): 608-613.

18. Chowdhury AH, Murray K, Hoad CL, Costigan C, Marciani L, et al. (2016) Effects of bolus and continuous nasogastric feeding on gastric emptying, small bowel water content, superior mesenteric artery blood flow, and plasma hormone concentrations in healthy adults: A randomized crossover study. Annals of surgery 263(3): 450-457.

19. Premji SS, Chessell L (2011) Continuous nasogastric milk feeding versus intermittent bolus milk feeding for premature infants less than 1500 grams. Cochrane Database of Systematic Reviews 9(11)

20. Aguilera Martinez R, Ramis Ortega E, Carratalá Munuera C, Fernández Medina JM, Saiz Vinuesa MD, et al. (2014) Effectiveness of continuous enteral nutrition versus intermittent enteral nutrition in intensive care patients: a systematic review. JBI Database of Systematic Reviews and Implementation Reports 12(1): 281-317.

21. Powers WJ, Rabinstein AA, Ackerson T, Adeoye OM, Bambakidis NC, et al. (2018) 2018 guidelines for the early management of patients with acute ischemic stroke: A guideline for healthcare professionals from the American Heart Association/American Stroke Association. Stroke 49(3): 46-110.

22. (2016) Royal college of Physicians. National clinical guideline for stroke Intercollegiate Stroke Working Party.

23. (2017) Stroke Foundation. Clinical Guidelines for Stroke Management.

24. Wang Y, Cai B (2011) Influnence of enteral nutrition via feeding pump based continuous infusion on nutrition support associated complication in stroke patients: Compared with conventional bolus infusion. Chinese Journal of Clinical Nutrition 19(1): 19-21.

25. Liberati A, Altman DG, Tetzlaff J, Mulrow C, Gøtzsche PC, et al. (2009) The PRISMA statement for reporting systematic reviews and meta analyses of studies that evaluate health care interventions: Explanation and elaboration. PLoS medicine 6(7):1000100.

26. Ortiz GA, L Sacco R (2014) National institutes of health stroke scale (nihss). Wiley Stats.

27. Schultz T, Florence Z (2007) Joanna Briggs institute meta-analysis of statistics assessment and review instrument.
28. París AS, Lázaro J, Guallar A, Gracia P, Caverni A, et al. (2005) Continuous enteral nutrition versus single bolus: Effects on urine $\mathrm{C}$ peptide and nitrogen balance. Medicina Clínica 124(16): 613-615.

29. Chen MA, Li LI, Wen LI, Wei J, Pan J, et al. (2017) Effects of continuous versus intermittent pump feeding on the energy intake in patients with acute stroke: A prospective randomized control trial. Chinese Journal of Clinical Nutrition 25(3): 153-158.

30. Güngör L, Özeke L, Türkel Y, Sandıkçı U (2011) The Comparison of Continuous and Intermittent Enteral Nutrition in Cerebrovascular Patients. Turkish Journal of Neurology 17(2): 76-82.

31. Sternbach GL (2000) The Glasgow coma scale. The Journal of emergency medicine 19(1): 67-71.

32. Knaus WA, Draper EA, Wagner DP, Zimmerman JE (1985) APACHE II: A severity of disease classification system. Critical care medicine 13(10): 818-829.

33. Mahoney FI, Barthel DW (1965) Functional evaluation: The Barthel Index: A simple index of independence useful in scoring improvement in the rehabilitation of the chronically ill. Maryland state medical journal.

34. Chen YC, Chou SS, Lin LH, Wu LF (2006) The effect of intermittent nasogastric feeding on preventing aspiration pneumonia in ventilated critically ill patients. The journal of nursing research: JNR 14(3): 167180.

35. MacLeod JB, Lefton J, Houghton D, Roland C, Doherty J, et al. (2007) Prospective randomized control trial of intermittent versus continuous gastric feeds for critically ill trauma patients. Journal of Trauma and Acute Care Surgery 63(1): 57-61.

36. Ciocon, Jerry O, Galindo Ciocon DJ, Tiessen C, Galindo D (1992) Continuous compared with intermittent tube feeding in the elderly. Journal of Parenteral and Enteral Nutrition 16(6): 525-528.

37. Lee JS, Kwok T, Chui PY, Ko FW, Lo WK, et al. (2010) Can continuous pump feeding reduce the incidence of pneumonia in nasogastric tube fed patients? A randomized controlled trial. Clinical Nutrition 29(4): 453-458.

38. Chen YC, Chou SS, Lin LH, Wu LF (2006) The effect of intermittent nasogastric feeding on preventing aspiration pneumonia in ventilated critically ill patients. The journal of nursing research: JNR 14(3): 167-80.

39. Sun WM, LA Houghton, NW Read, DG Grundy, AG Johnson (1988) Effect of meal temperature on gastric emptying of liquids in man. Gut 29(3): 302-305.

40. Mishima Yuko, Amano Y, Takahashi Y, Mishima Y, Moriyama N, et al. (2009) Gastric emptying of liquid and solid meals at various temperatures. Journal of gastroenterology 44(5): 412-418.

41. Webber DE, M Nouri, FR Bell (1980) A study of the effects of meal temperature on gastric function. Pflügers Archiv 384(1): 65-68.

42. Bateman DN (1982) Effects of meal temperature and volume on the emptying of liquid from the human stomach. The Journal of physiology 331(1): 461-467.

43. Hiebert John M, Brown A, Anderson RG, Halfacre S, Rodeheaver GT, et al. (1981) Comparison of continuous vs intermittent tube feedings in adult burn patients. Journal of Parenteral and Enteral Nutrition 5(1): 73-75.

44. Maeve Tavares de Araujo, Viviane, Paulo César Gomes, Cervantes Caporossi (2014) Enteral nutrition in critical patients should the administration be continuous or intermittent? Nutricion hospitalaria 29(3): 563-567.

45. Kadamani Ibrahim, Itani M, Zahran E, Taha N (2014) Incidence of aspiration and gastrointestinal complications in critically ill patients using continuous versus bolus infusion of enteral nutrition: A pseudo randomised controlled trial. Australian Critical Care 27(4): 188-193.

46. Nasiri Morteza, Farsi Z, Ahangari M, Dadgari F (2017) Comparison of Intermittent and Bolus Enteral Feeding Methods on Enteral Feeding 
Intolerance of Patients with Sepsis: A Triple blind Controlled Trial in Intensive Care Units. Middle East journal of digestive diseases 9(4): 218227.

47. Abdelsalam Yousry (2012) Continuous versus bolus infusion of enteral nutrition in intensive care unit. AAMJ 10(3).

48. Serpa Letícia Faria, Kimura M, Faintuch J, Ceconello I (2003) Effects of continuous versus bolus infusion of enteral nutrition in critical patients. Revista do Hospital das Clinicas 58(1): 9-14.

49. Marathe Chinmay S, Rayner CK, Jones KL, Horowitz M (2013) Relationships between gastric emptying, postprandial glycemia, and incretin hormones. Diabetes care 36(5): 1396-1405.
50. Plummer Mark P, Karen L Jones, Caroline E Cousins, Laurence G, et al. (2015) Hyperglycemia potentiates the slowing of gastric emptying induced by exogenous GLP-1. Diabetes Care 38(6): 1123-1129.

51. Schvarcz E, Palmér M, Aman J, Horowitz M, Stridsberg M, et al. (1997) Physiological hyperglycemia slows gastric emptying in normal subjects and patients with insulin dependent diabetes mellitus. Gastroenterology 113(1): 60-66.

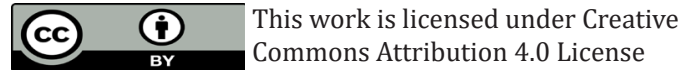

To Submit Your Article Click Here: Submit Article

DOI: $10.32474 / 0 J N B D .2019 .03 .000163$

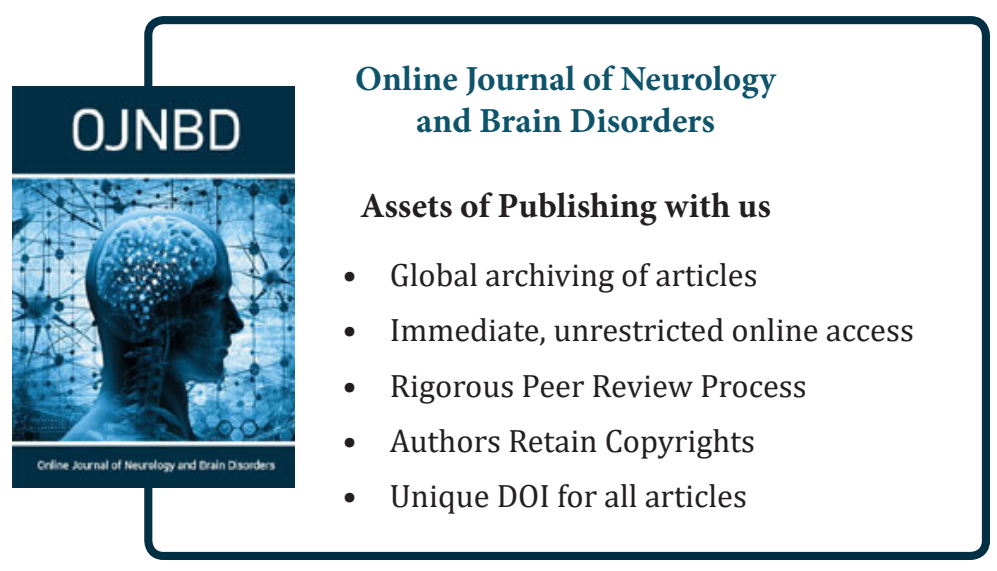

